\title{
Diffusion dans les liquides
}

\author{
A.J. Dianoux \\ Institut Laue-Langevin, 6 rue Jules Horowitz, BP. 159, 38042 Grenoble cedex 9, France
}

\begin{abstract}
Résumé : Après une brève introduction qui rappelle les concepts détaillés dans le cours de M. Bée, nous présentons un aperçu de trois de nos travaux sur l'étude de la diffusion. Tout d'abord la dynamique de l'eau, dans son état normal ou surfondu, révèle la complexité apportée par le réseau de liaisons hydrogène. Ensuite l'effet du confinement sur la dynamique de l'eau sera étudié dans le cas de la membrane Nafion. Enfin la diffusion dans les phases nématique et smectique A d'un cristal liquide permet d'obtenir la valeur du potentiel qui maintient les couches dans la phase smectique.
\end{abstract}

\section{1) Introduction}

Le lecteur est renvoyé au cours de M. Bée pour une présentation détaillée de la fonction de diffusion des neutrons dans le cas d'une dynamique translationnelle continue ou par sauts. Nous rappellerons rapidement la loi de Fick et la relation d'Einstein. Puis nous présenterons le cas de la diffusion unidimensionnelle, pour une diffusion normale ou à la file indienne. Enfin le cas de la diffusion cohérente de particules se déplaçant par sauts sur un réseau de Bravais sera décrit. Un ouvrage de référence pour l'étude des liquides par la diffusion neutronique est celui d'Egelstaff [1].

\section{1 - Loi de Fick}

Cette loi, énoncée en 1855, dit que le flux est proportionnel au gradient de concentration dans une direction donnée :

$$
J_{x}=-D_{x} \frac{d c}{d x}
$$

La seconde loi de Fick dérive d'une équation de continuité (conservation de matière) :

$$
\frac{\partial \mathrm{c}}{\partial \mathrm{t}}=-\nabla \cdot \overrightarrow{\mathrm{J}}
$$

Dans le cas où le coefficient de diffusion est une constante, on obtient :

$$
\frac{\partial c}{\partial t}=D \nabla^{2} \mathrm{c}
$$


La fonction de Van Hove (pour une diffusion incohérente) se relie directement à la concentration par unité de volume :

$$
G_{s}(\vec{r}, t)=\frac{c(\vec{r}, t)}{N}
$$

La solution de (3) et (4) est :

$$
G_{s}(\vec{r}, t)=\frac{1}{(4 \pi D t)^{3 / 2}} \exp \left(-\frac{r^{2}}{4 D t}\right)
$$

Par double transformée de Fourier (spatiale et temporelle), on obtient la loi de diffusion :

$$
\mathrm{S}_{\mathrm{inc}}(\overrightarrow{\mathrm{Q}}, \omega)=\frac{1}{\pi} \frac{\mathrm{DQ}^{2}}{\left(\mathrm{DQ}^{2}\right)^{2}+\omega^{2}}
$$

\section{2 - Relation d'Einstein}

Pour la diffusion par sauts le lecteur pourra consulter le cours de M. Bée.

Le déplacement carré moyen s'exprime par :

$$
\left\langle r^{2}(t)\right\rangle=\int r^{2} G_{s}(\vec{r}, t) d \vec{r}=6 D t
$$

La dernière égalité s'obtient pour une diffusion continue, en utilisant l'équation (5).

Pour une marche au hasard dans un réseau cubique où 1 est la distance de sauts et $\tau$ le temps entre sauts, la relation d'Einstein relie les sauts à l'échelle atomique au coefficient de diffusion macroscopique :

$$
\mathrm{D}=\frac{\mathrm{1}^{2}}{6 \tau}
$$

\section{3 - Diffusion unidimensionnelle (1D)}

C'est le cas extrême d'une diffusion anisotrope pour laquelle deux cas différents peuvent se produire : si les molécules dans un canal donné peuvent se croiser, on a alors la diffusion 1D normale (Figure 1). Si le rayon du canal est inférieur au diamètre d'une molécule, il n'y a pas de croisement possible et les molécules doivent garder le même ordre : c'est la diffusion à la file indienne. 


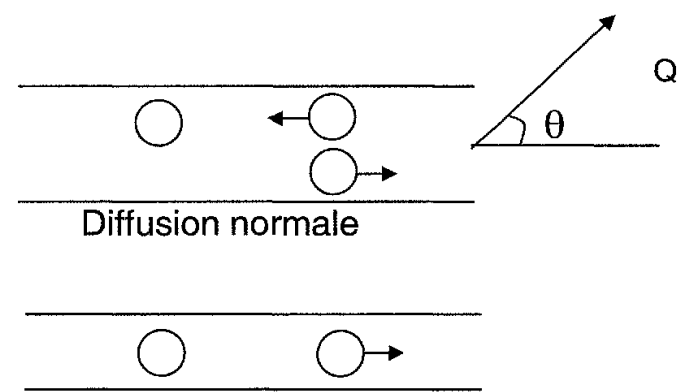

Diffusion à la file indienne

Fig. 1 : Schéma pour une diffusion unidimensionnelle normale ou à la file indienne

\subsection{1 - Diffusion normale}

Si on regarde dans une direction $\theta$, inclinée par rapport à l'axe du canal, la loi de diffusion unidimennsionnelle normale devient [2] :

$$
\mathrm{S}_{\mathrm{ID}}(\overrightarrow{\mathrm{Q}}, \omega)=\frac{1}{\pi} \frac{\mathrm{DQ}^{2} \cos ^{2} \theta}{\left(\mathrm{DQ}^{2} \cos ^{2} \theta\right)^{2}+\omega^{2}}
$$

Si l'échantillon est constitué de canaux non orientés, il faut moyenner l'équation (9) :

$$
S_{1 D}(Q, \omega)=\frac{1}{2 \pi} \int_{0}^{\pi} d \theta \frac{D Q^{2} \cos ^{2} \theta \sin \theta}{\left(D Q^{2} \cos ^{2} \theta\right)^{2}+\omega^{2}}
$$

L'expression résultante a été calculée par Jobic et al [3]. La fonction obtenue est plus pointue qu'une lorentzienne ayant la même largeur à mi-hauteur.

\subsection{2 - Diffusion à la file indienne}

Le déplacement carré moyen n'est plus donné par l'équation (7), mais par [4] :

$$
\sigma^{2}=2 \mathrm{Ft}^{1 / 2}
$$


où $\mathrm{F}$ est le facteur de mobilité.

La fonction de diffusion intermédiaire est alors :

$$
\mathrm{I}_{\mathrm{Fl}}(\mathrm{Q}, \mathrm{t})=\exp \left(-\mathrm{FQ}^{2} \mathrm{t}^{1 / 2}\right)
$$

La fonction de diffusion $\mathrm{S}_{\mathrm{FI}}(\mathrm{Q}, \omega)$ s'exprime par des intégrales de Fresnel [5].

\section{4 - Diffusion cohérente}

Pour des particules diffusant sur un réseau de Bravais, le modèle de Chuddey-Elliott donne la largeur de la lorentzienne de diffusion, dans le cas de la diffusion incohérente :

$$
\begin{aligned}
& \Gamma_{i n c}(\vec{Q})=\frac{1}{n \tau(c)} \sum_{i=1}^{n}\left(1-\exp \left(-\vec{Q} \cdot \vec{r}_{i}\right)\right) \\
& \operatorname{avec} \tau(\mathrm{c})=\tau(0) /(1-\mathrm{c})
\end{aligned}
$$

Le blocage de site réduit la largeur incohérente d'un facteur (1-c), mais ceci n'est valable que dans la limite de faible concentration.

La fonction de diffusion cohérente est donnée par [6] :

$$
\mathrm{S}_{\text {coh }}(\overrightarrow{\mathrm{Q}}, \omega)=(1-\mathrm{c}) \mathrm{S}_{\mathrm{inc}}(\overrightarrow{\mathrm{Q}}, \omega)
$$

Toutefois sa largeur est indépendante de la concentration :

$$
\Gamma_{\mathrm{coh}}(\overrightarrow{\mathrm{Q}}, \mathrm{c})=\lim _{\mathrm{c} \rightarrow \mathrm{o}}\left[\Gamma_{\mathrm{inc}}(\overrightarrow{\mathrm{Q}}, \mathrm{c})\right]
$$

Les effets de blocage de site et de corrélation s'annulent en moyenne. La généralisation [7] pour tenir compte des corrélations entre particules, dans la limite du champ moyen, donne une largeur $\Gamma_{\text {coh }}^{\prime}(\vec{Q})$ :

avec

$$
\Gamma_{c o h}^{\prime}(\vec{Q})=\frac{\Gamma_{c o h}(\vec{Q})}{S(\vec{Q})}
$$


$S(\vec{Q})$ est la fonction de diffusion diffuse. Elle a ,en général, des pics larges à des positions où apparaissent des pics de Bragg quand le système est ordonné. La largeur de raie diminue donc là où il y a un pic de $S(\vec{Q})$.

Ce résultat avait été donné par de Gennes [8] en utilisant des arguments généraux.

Nous allons maintenant exposer trois de nos travaux portant sur la diffusion. Tout d'abord sera présenté le cas de la diffusion dans un liquide apparemment simple, l'eau. Ensuite nous verrons l'effet d'un confinement ; ceci sera appliqué à la diffusion de l'eau dans la membrane Nafion. Enfin sera exposée la diffusion dans les phases smectique et nématique d'un cristal liquide.

\section{2) Diffusion dans l'eau (normale et surfondue)}

L'eau est un liquide moléculaire simple, mais sa dynamique est complexe, due au réseau fluctuant de liaisons hydrogène. L'interprétation de spectres quasi-élastiques des neutrons est donc sujette à caution si on se restreint à une gamme étroite autour de la température ambiante. J. Teixeira et al [9] ont déjà montré que le réseau de liaisons hydrogène devient de plus en plus étendu lorsque la température décroît en dessous de $0^{\circ} \mathrm{C}$ (eau surfondue). Ceci permet de séparer deux échelles de temps associées aux mouvements diffusifs microscopiques des molécules d'eau. Cette étude a été reprise avec le spectromètre IN6 de l'ILL qui a permis d'étudier cette dynamique dans une large gamme de température et de transfert de moment.

\section{1 - Séparation des mouvements}

Nous faisons l'hypothèse simplificatrice qui est exposée dans le cours de M. Bée. Le modèle de Sears [10] donne la loi de diffusion intermédiaire associée à la rotation de la molécule d'eau :

$$
R(Q, t)=j_{0}^{2}(Q a)+j_{1}^{2}(Q a) \exp \left(-t / 3 \tau_{1}\right)+5 j_{2}^{2}(Q a) \exp \left(-t / \tau_{i}\right)+\ldots
$$

où $\tau_{1}$ est le temps de relaxation de la diffusion rotationnelle; $a=0.98 \AA$ (distance $\mathrm{O}-\mathrm{H}$ ). Ceci permet de déterminer la largeur translationnelle [1] :

$$
\Gamma(Q)=\frac{D Q^{2}}{1+D Q^{2} \tau_{o}}
$$

où $\tau_{o}$ est le temps de résidence dans une configuration donnée de liaisons hydrogène. La relation d'Einstein (Eq. 8) permet de donner une distance de sauts moyenne en fonction du coefficient de diffusion :

$$
\left\langle\ell^{2}\right\rangle_{\mathrm{av}}^{1 / 2}=6 \tau_{\mathrm{o}} \mathrm{D}
$$




\section{2 - Méthode expérimentale [11]}

L'échantillon était constitué de 80 tubes en pyrex de $0.1 \mathrm{~mm}$ d'épaisseur et de diamètre intérieur de $0.3 \mathrm{~mm}$. L'assemblage des tubes permet de couvrir une surface de $5 \times 5 \mathrm{~cm}^{2}$. L'utilisation du pyrex (qui contient du bore) a conduit à une diffusion multiple inférieure à $5 \%$ grâce à l'absorption qui a lieu dans les parois en pyrex. Nous avons pris des spectres à 9 températures : $20,12,5,-5,-10,-12,-17$ et $-20^{\circ} \mathrm{C}$.

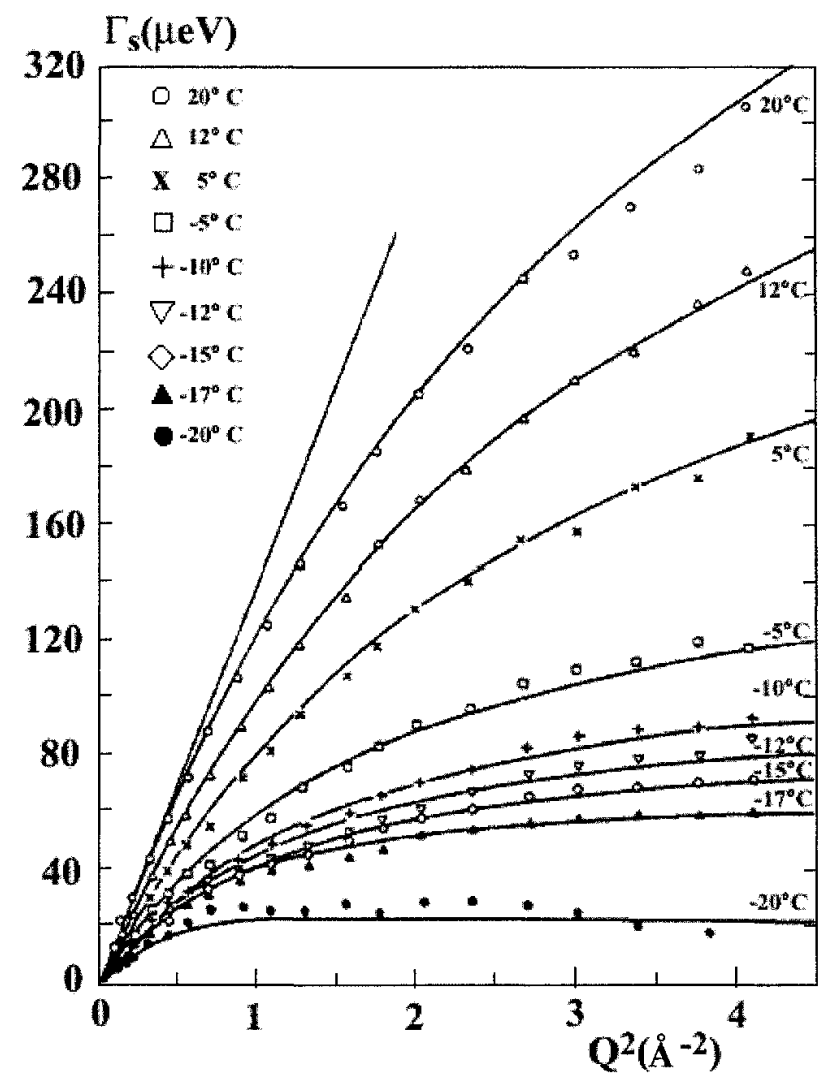

Fig. 2: Largeur de raie $\Gamma$ de la composante translationnelle des spectres de l'eau en fonction de $\mathrm{Q}^{2}$. Il faut noter que l'ajustement avec une seule lorentzienne donne des largeurs beaucoup plus grandes, avec une dépendance en $Q$ différente. Les lignes en traits pleins représentent l'ajustement avec Eq. 19. La ligne droite donne le coefficient de diffusion à $20^{\circ} \mathrm{C}$. 


\section{3 - Résultats}

Le temps caractéristique de la rotation $\tau_{1}$ suit bien une loi d'Arrhénieus donnée par :

$$
\tau_{1}=\tau_{1}^{0} \exp \left(\mathrm{E}_{\mathrm{A}} / \mathrm{k}_{\mathrm{B}} \mathrm{T}\right)
$$

avec une énergie d'activation $\mathrm{E}_{\mathrm{A}}=1.85 \mathrm{kcal}^{\mathrm{mol}}{ }^{-1}$

En utilisant cette loi, nous avons pu extraire à chaque angle et à chaque température la largeur translationnelle $\Gamma(Q)$ qui est reportée sur la figure 2. En utilisant l'équation 19, on a pu tirer les valeurs de $\mathrm{D}$ et de $\tau_{\mathrm{o}}$ pour chaque température. Il faut noter que la mesure de $\mathrm{D}$ à basse température n'est pas très précise, puisque $\mathrm{D}$ est déterminé à partir de $\Gamma(\mathrm{Q})$ aux faibles valeurs de $Q$ où la largeur de raie est plus faible que la résolution du spectromètre. Dans le tableau I, nous avons reporté l'ensemble des paramètres en fonction de la température.

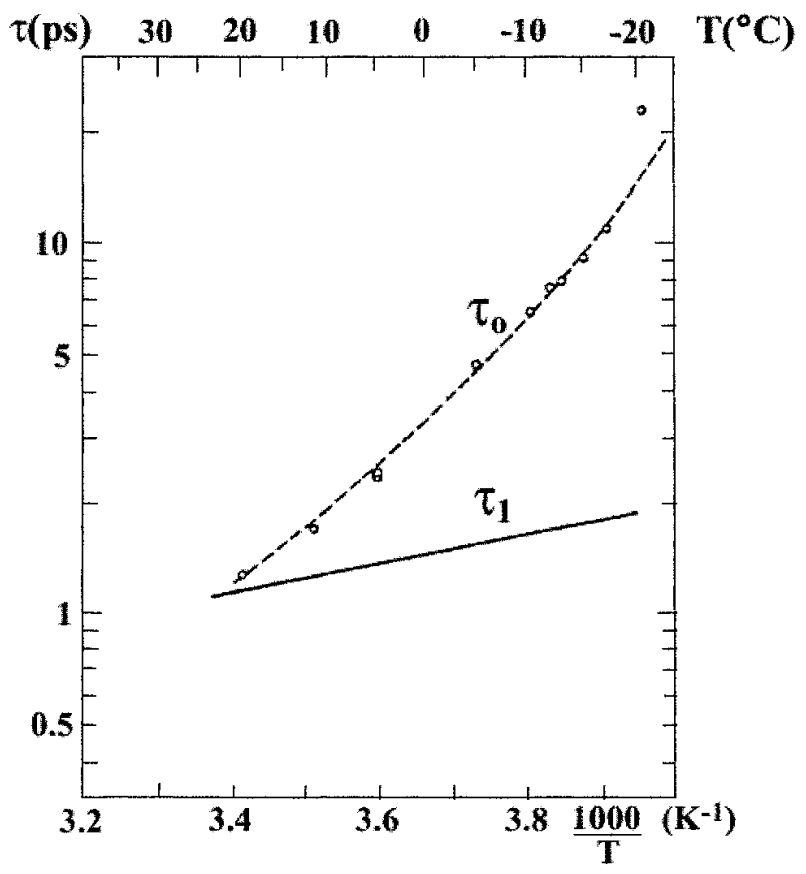

Fig. $3:$ Temps de résidence $\tau_{o}$ pour la diffusion translationnelle et tracé d'Arrhénius du temps de relaxation $\tau_{1}$ de la diffusion rotationnelle. 
La distance moyenne de saut $L=\left\langle\ell^{2}\right\rangle^{1 / 2}$ est obtenue à partir de l'équation (20), en utilisant les valeurs de $\mathrm{D}$ mesurées par RMN. Finalement la figure 3 présente la variation en fonction de la température des deux temps $\tau_{\mathrm{o}}$ et $\tau_{1}$. On voit que à température ambiante ces deux temps sont très proches, ce qui explique la difficulté d'analyser correctement les spectres. Dans le domaine surfondu, ces deux temps se séparent : le temps caractéristique rotationnel suit une loi d'Arrhénius, alors que le temps $\tau_{\mathrm{o}}$ diverge fortement à basse température. Ce temps $\tau_{0}$ est associé au mouvement d'ensemble de la molécule d'eau et sa variation est similaire à celles déterminées à partir d'autres propriétés de transport [12].

Enfin dans le tableau I est reportée la valeur $\left\langle\mathrm{u}^{2}\right\rangle^{1 / 2}=0.48 \AA$ pour le déplacement carré moyen dû aux vibrations des hydrogènes. Ceci correspond à un angle critique de $30^{\circ}$ pour lequel il y a rupture de la liaison hydrogène.

TABLE I. Paramètres pour chaque température

\begin{tabular}{|c|c|c|c|c|}
\hline$T\left({ }^{\circ} \mathrm{C}\right)$ & $\tau_{0}(\mathrm{ps})$ & $\tau_{1}(\mathrm{ps})$ & $L(\AA)$ & $\left\langle u^{2}\right\rangle^{1 / 2}(\AA)$ \\
\hline 20 & 1.25 & & 1.29 & \\
\hline 12 & 1.66 & & 1.25 & \\
\hline 5 & 2.33 & $0.0485 \exp \left(E_{A} / k_{B} T\right)$ & 1.32 & \\
\hline-5 & 4.66 & & 1.54 & \\
\hline-10 & 6.47 & $E_{\mathrm{A}}=1.85 \mathrm{kcal} \mathrm{mol}^{-1}$ & 1.65 & 0.48 \\
\hline-12 & 7.63 & & 1.70 & \\
\hline-15 & 8.90 & & 1.73 & \\
\hline-17 & 10.8 & & 1.80 & \\
\hline-20 & 22.7 & & 2.39 & \\
\hline
\end{tabular}

\section{3) Diffusion dans un milieu confiné}

La diffusion de molécules dans des géométries restreintes se retrouve dans une grande variété de situations physiques. Récemment un colloque a eu lieu à l'ILL sur ce thème [13].

Le modèle le plus simple d'un milieu confiné est celui d'une sphère.

\section{1 - Modèle de diffusion dans une sphère [14]}

L'équation gouvernant l'évolution de la fonction de Van Hove pour une particule diffusant dans un potentiel $\mathrm{V}(\overrightarrow{\mathrm{r}})$ est : 


$$
\frac{\partial G_{s}}{\partial t}=\nabla \cdot D \cdot \nabla G_{S}+\frac{1}{k_{B} T} \nabla \cdot\left[(D \cdot \nabla V) G_{S}\right]
$$

Pour un potentiel de diffusion sphérique, on a :

$$
\begin{aligned}
& V(r)=0 \text { pour } 0<r<a \text { (rayon de la sphère) } \\
& V(r)=+\infty \text { pour } r>a
\end{aligned}
$$

Le facteur de structure incohérent élastique est donné par :

$$
\mathrm{A}_{0}^{0}(Q)=\left[\frac{3 j_{i}(Q a)}{Q a}\right]^{2}
$$

La loi de diffusion est donc :

$$
S_{\text {inc }}(Q, \omega)=A_{0}^{0}(Q) \delta(\omega)+\sum \text { lorentziennes }
$$

Ces lorentziennes ont une largeur proportionnelle à $\mathrm{D} / \mathrm{a}^{2}$. Pour $\mathrm{Q} \rightarrow 0$, la largeur tend vers $4.333 \mathrm{D} / \mathrm{a}^{2}$. La figure 4 donne la variation de la largeur de raie en fonction de $(\mathrm{Qa})^{2}$. On retrouve que pour $\mathrm{Qa}>\pi$, la largeur de raie varie pratiquement comme $(\mathrm{Qa})^{2}$.

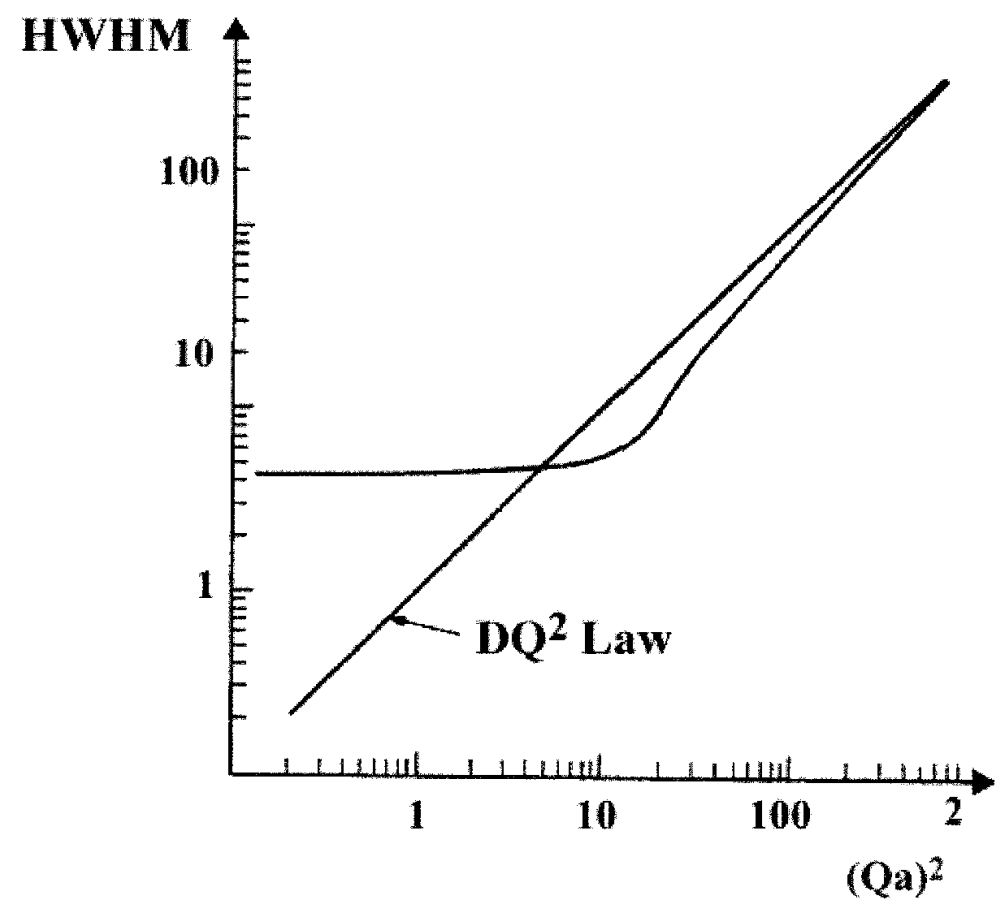

Fig. 4: Variation de la demi-largeur à mi-hauteur (HWHM) de la composante élargie des spectres pour une diffusion à l'intérieur d'une sphère de rayon a. L'échelle de largeur est en unités de $\mathrm{D} / \mathrm{a}^{2}$, en fonction de (Qa) ${ }^{2}$. 


\section{2 - Diffusion de l'eau dans la membrane Nafion [15]}

La membrane Nafion a une chaîne principale perfluorée avec des groupements latéraux acides. Elle peut absorber beaucoup d'eau ( $\sim 15 \%$ en poids). Grâce à sa résistance chimique, sa conductivité ionique et sa sélectivité à la perméation, ces membranes sont beaucoup utilisées dans les batteries d'électrolyse.

Les spectres quasi-élastiques ont eté enregistrés avec le spectromètre IN5 à 10,11 et $13 \AA$ (résolutions de 18.5, 14 et $9 \mu \mathrm{eV}$ ). La largeur de raie de la composante quasi-élastique est rapportée sur la figure 5. Sa variation en fonction de $\mathrm{Q}^{2}$ se rapproche beaucoup de celle de la figure 4.

L'analyse des spectres en terme du modèle, développée dans la référence [15], conduit à la valeur du coefficient $\mathrm{D}=1.8 \times 10^{-5} \mathrm{~cm}^{2} / \mathrm{s}$ (à $25^{\circ} \mathrm{c}$ ) et à un rayon de confinement $\mathrm{a}=4.25 \AA$. La diffusion à longue distance a été mesurée par marquage radioactif avec du tritium. Elle est beaucoup plus petite $\left(D_{t}=1.6 \times 10^{-6} \mathrm{~cm}^{2} / \mathrm{s}\right)$, mais a une influence non négligeable pour ajuster correctement la partie centrale du spectre, comme cela est montré sur la figure 6 .

Le modèle de diffusion dans une sphère a été étendu au cas d'un cylindre, pour obtenir un volume avec une forme anisotrope [16]. Nous avons montré que la diffusion de l'eau dans un échantillon de Nafion étiré reste isotrope dans un volume de l'ordre de $10 \AA ̊$ de diamètre.

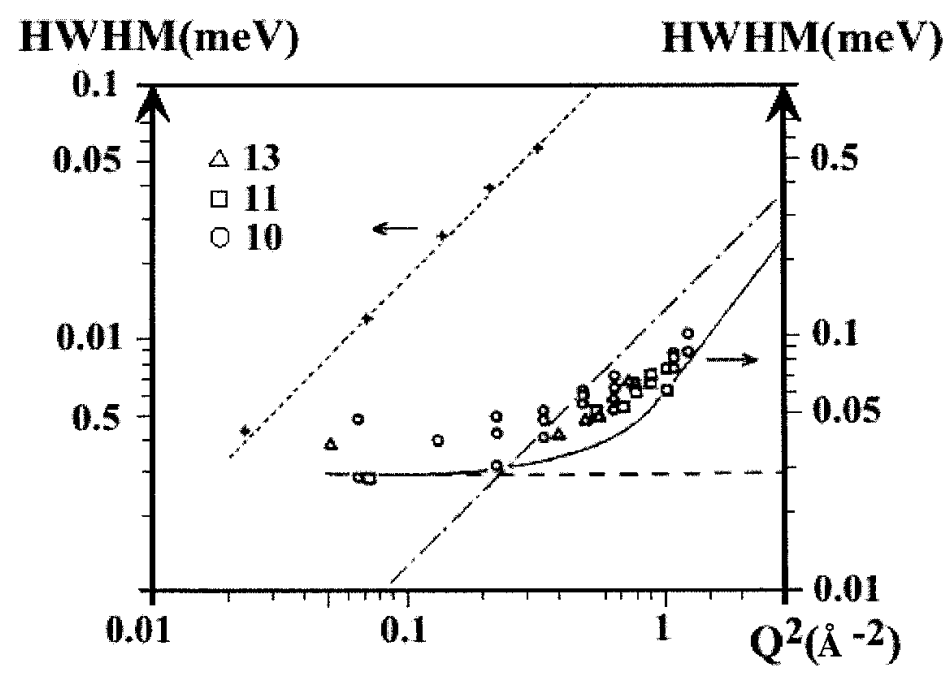

Fig. 5 : Demi-largeur à mi-hauteur de la composante élargie des spectres obtenus avec la membrane Nafion contenant $15 \%$ en poids d'eau, à une température de $25^{\circ} \mathrm{c}$. Les points sont les largeurs obtenues par l'ajustement avec une lorentzienne: $(0)$ en utilisant une largeur d'onde de $10 \AA,(\square)$ de $11 \AA,(\Delta)$ de $13 \AA$. La ligne en trait plein est la valeur théorique prédite par le modèle de diffusion dans une sphère avec $\mathrm{D}=1.8 \times 10^{-5} \mathrm{~cm}^{2} / \mathrm{s}$ et a $=4.25 \AA$. Les deux asymptotes théoriques pour $\mathrm{Q} \rightarrow 0$ et $\mathrm{Q} \rightarrow \infty$ sont tracées. $(+)$ demi-largeur à mihauteur obtenue par l'ajustement avec une lorentzienne pour les spectres mesurés dans l'eau en masse à une température de $28^{\circ} \mathrm{C}$ (longueur d'onde incidente de $10 \AA$ ). La ligne pointillée passant par les points $(+)$ est la largeur prédite par le modèle de diffusion simple avec $D=2.5$ $\times 10^{-5} \mathrm{~cm}^{2} / \mathrm{s}$. Prière de noter les échelles verticales différentes pour les échantillons Nafion et eau massique. 


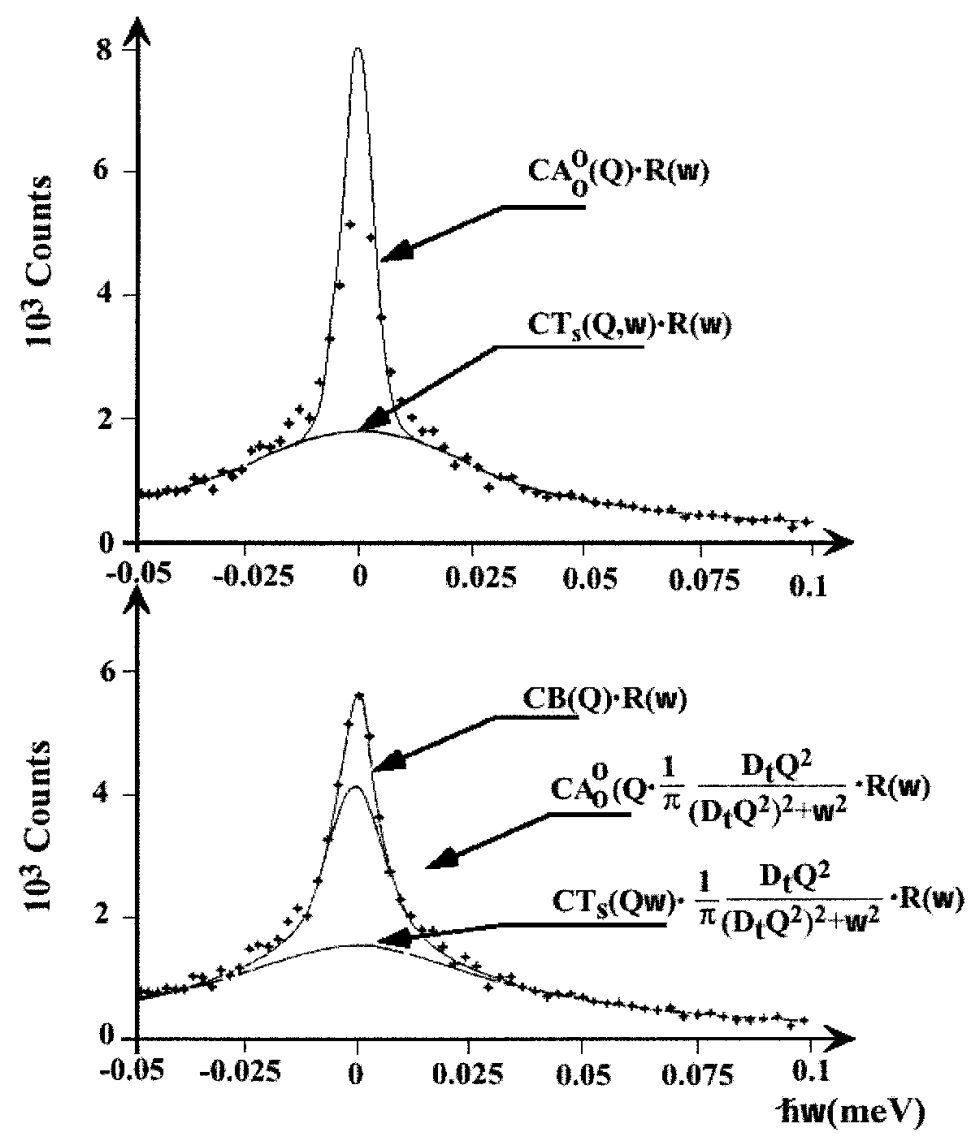

(a)

(b)

Fig. 6 : Spectre quasi élastique pour $Q=0.635 \AA^{-1}$ obtenu avec une longueur d'onde incidente de $13 \AA$. Dans (a), l'ajustement est obtenu avec le modèle de diffusion dans une sphère avec $\mathrm{D}=1.8 \times 10^{-5} \mathrm{~cm}^{2} / \mathrm{s}$ et a $=4.25 \AA$; il n'y a pas de composante élastique dûe à la membrane ; noter le mauvais ajustement autour de la région élastique.

Dans (b), il est tenu compte du coefficient de diffusion à grande distance $D_{t}=1.6 \times 10^{-6} \mathrm{~cm}^{2} / \mathrm{s}$ et d'une contribution élastique dûe à la membrane.

\section{4) Diffusion en présence d'un potentiel sinusoïdal}

Le problème de la diffusion dans des potentiels périodiques se trouve dans une grande variété de situations physiques, en particulier la diffusion des molécules à travers les couches dans les phases smectiques des cristaux liquides. 


\section{1- Théorie [17]}

La particule diffuse à une dimension (suivant $z$ ) en présence d'un potentiel sinusoïdal :

$$
\mathrm{V}_{\mathrm{N}}(\mathrm{z})=-\frac{\mathrm{V}_{\mathrm{N}}}{2} \cos \left(\mathrm{Nq}_{\mathrm{c}} \mathrm{z}\right)
$$

où $q_{c}=\frac{2 \pi}{a}$ (a est la distance entre couches).

La forme simplifiée de l'équation 22, s'écrit :

$$
\begin{aligned}
& \frac{\partial G_{s}}{\partial t}=D_{o} \frac{\partial}{\partial z}\left[\frac{\partial G_{s}}{\partial z}+N \gamma_{N} q_{c} \sin \left(N q_{c} z\right) \cdot G_{s}\right] \\
& \operatorname{avec}_{\gamma_{N}}^{\prime}=\frac{V_{N}}{2 k_{B} T}
\end{aligned}
$$

Pour $\mathrm{N}=1$, on obtient l'équation gouvernant la diffusion dans une phase smectique. La solution n'est pas analytique, mais peut être résolue sous forme matricielle. En particulier, le coefficient de diffusion est donné par :

$$
\mathrm{D}_{\mathrm{t}}=\mathrm{D}_{\mathrm{o}} / \mathrm{I}_{\mathrm{o}}^{2}\left(\gamma_{1}^{\prime}\right)
$$

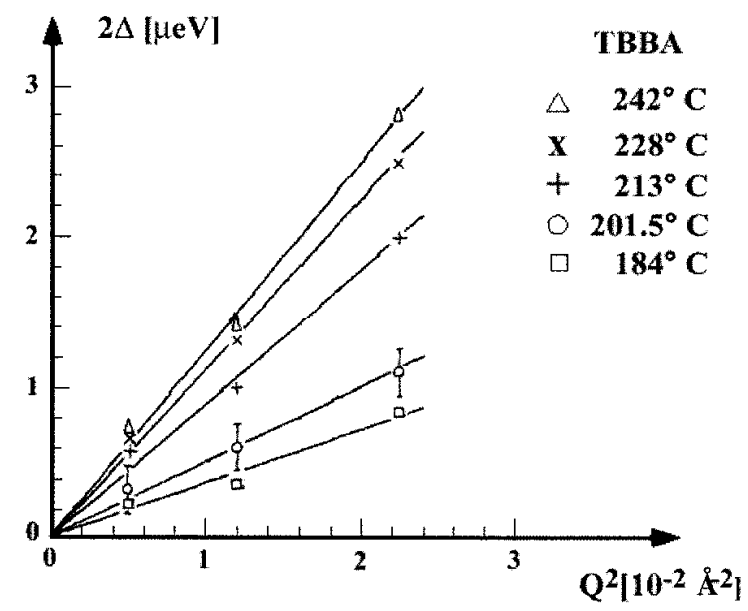

Fig. 7 : Largeur à mi-hauteur $2 \Delta$ en fonction de $Q^{2}$ pour la diffusion dans le cristal liquide TBBA, à différentes températures. La pente des lignes droites donne la valeur du coefficient de diffusion moyen $\bar{D}$ dans les phases isotrope, smectique $A$ et nématique.

\section{2 - Application à la diffusion dans un cristal liquide, le TBBA}

Par la technique de rétro-diffusion, nous avons mesuré les coefficients d'autodiffusion moyens dans les phases smectique A, nématique et isotrope du TBBA [18].

Les mesures ont été faites sur IN10, à de très petits $Q: 0.07,0.11$ et $0.15 \AA^{-1}$. Dans ces conditions la largeur quasi-élastique est :

$$
2 \Delta=2 \overline{\mathrm{D}} \mathrm{Q}^{2}
$$




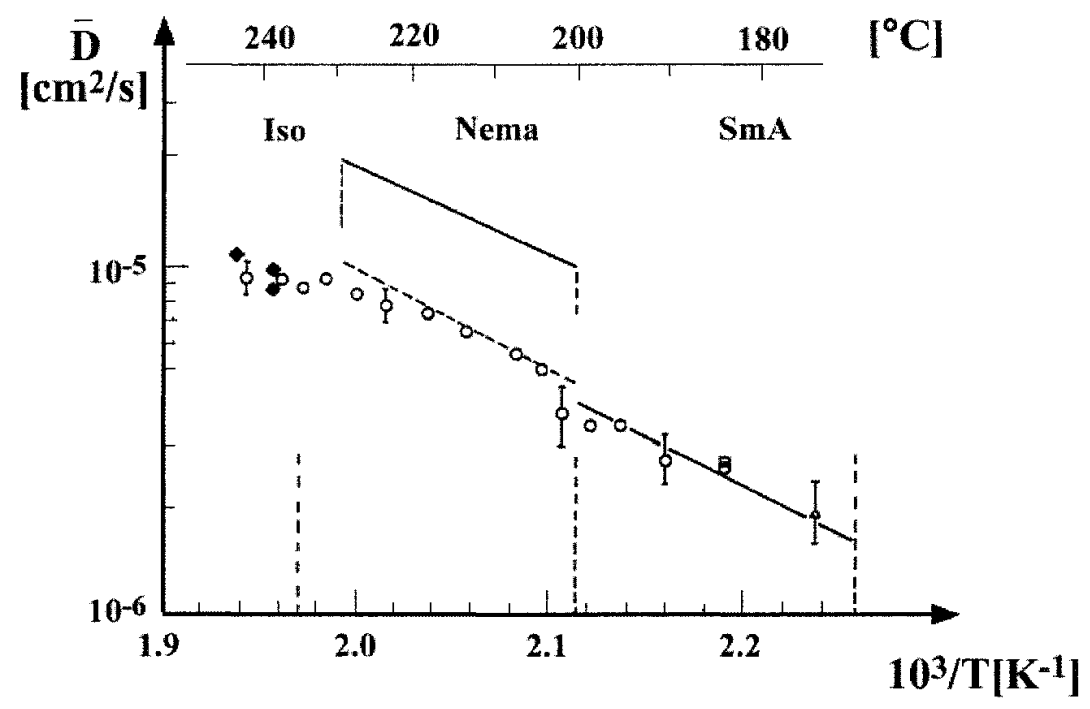

Fig. 8: Coefficients de diffusion moyens $\bar{D}$ du TBBA en fonction de l'inverse de la température, dans les phases smectique $\mathrm{A}$, nématique et isotrope. Les symboles ouverts se réfèrent à des travaux avec les neutrons; 0 : ce travail, $\square$ et $\Delta$ : deux points mesurés précédemment. Lignes en traits pleins : valeur moyenne déduite des mesures RMN dans la phase smectique $A$ [19] et la phase nématique [20]; $\bullet$ valeur RMN dans la phase isotrope [19]. La ligne pointillée donne la valeur de $\bar{D}$ dans la phase nématique en utilisant le modèle de diffusion dans un potentiel périodique pour la phase smectique $A$. On voit que ce modèle prédit bien une très faible discontinuité au passage smectique $\mathrm{A}$ - nématique, à cause de la faible valeur du potentiel $\mathrm{V}$ dans la phase smectique.

Nous mesurons le coefficient moyen $\overline{\mathrm{D}}$, car l'échantillon ne peut pas être orienté sur une si grande surface (Fig.7). Ce résultat est comparé avec des résultats RMN [19] pour les phases smectique A et isotrope, et d'autres résultats RMN [20] pour la phase nématique.

Le coefficient moyen est donné par :

$$
\overline{\mathrm{D}}=\frac{1}{3} \mathrm{D}_{11}+\frac{2}{3} \mathrm{D}_{\perp}
$$

La figure 8 montre qu'il y a un bon accord avec les données RMN pour les phases smectique A et isotrope, mais un violent désaccord (facteur de deux) pour la phase nématique. Les données neutrons prouvent que cette méthode RMN qui utilise une séquence particulière à 
deux pulses ( « Nematic echo ») donne des résultats trop élevés. Le modèle présenté dans la section précédente permet de calculer le potentiel $V$ qui maintient les couches smectiques. Dans les phases nématique et smectique on a :

$$
D_{\perp}(T)=A_{\perp} \exp \left(-\frac{E_{\perp}}{k_{B} T}\right)
$$

où $A_{\perp}$ est une facteur d'échelle et $E_{\perp}$ l'énergie d'activation pour la diffusion perpendiculaire au directeur.

Dans la phase nématique on a de même :

$$
D_{\|}^{\text {nem }}(T)=A_{\|} \exp \left(-\frac{E_{\|}}{k_{B} T}\right)
$$

Dans la phase smectique A, il y a un potentiel V qui tient les couches. Suivant l'équation (26) on a :

$$
D_{11}^{\text {smA }}=A_{\| 1} \exp \left(-\frac{E_{\|}}{k_{B} T}\right) / I_{o}^{2}\left(\frac{V}{2 k_{B} T}\right)
$$

En ajustant les courbes de $\overline{\mathrm{D}}$ dans les phases nématique et smectique $\mathrm{A}$, on calcule un potentiel $\mathrm{V}=1.1 \mathrm{kcal} / \mathrm{mole}$ qui est en bon accord avec les estimations théoriques et des mesures des rayons $X$ sur le déplacement carré moyen suivant la normale aux plans smectiques.

\section{Références}

[1] Egelstaff P.A., An introduction to the liquid state (Academic Press, London, 1967)

[2] Dianoux A.J., Volino F., Hervet H., Mol. Phys. 30 (1975) 1181

[3] Jobic H., Bée M., Renouprez A., Surf. Sci. 140 (1984) 307

[4] Hahn K., Kärger J., J. Phys. A 28 (1995) 3061

[5] Jobic H., J. Physique IV 10, Pr 1 (2000) 77

[6] Bull D.J., Ross D.K., Physica B 301 (2001) 54

[7] Sinha S.K., Ross D.K., Physica B 149 (1988) 61

[8] De Gennes P.G., Physica 25 (1959) 825

[9] Chen S.H., Teixeira J., Nicklow R., Phys. Rev. A 26 (1982) 3477

[10] Sears V.F., Can J. Phys. 44 (1966) 1299 ; 45 (1966) 237

[11] Teixeira J., Bellissent-Funel M.C., Chen S.H., Dianoux A.J., Phys. Rev. A 31 (1985) 1913

[12] Angell C.A., Ann. Rev. Phys. Chem. 34 (1983) 593

[13] Frick B., Zorn R., Büttner H., Eds, J. Physique IV 10, Pr7 (2000)

[14] Volino F., Dianoux A.J., Mol. Phys. 41 (1980) 271

[15] Volino F., Pinéri M., Dianoux A.J., J. Polymer Sc., Polymer Physics Ed. 20 (1982) 481

[16] Dianoux A.J., Pinéri M., Volino F., Mol. Phys. 46 (1982) 129

[17] Volino F., Dianoux A.J., Mol. Phys. 36 (1978) 389

[18] Volino F., Dianoux A.J., Heidemann A., J. Physique lettres 40 (1979) L-583

[19] Krüger G.J., Spiesecke H., Van Steenwaikel R., J. Physique Colloq. 37 (1976) C3-123

[20] Krüger G.J., Weiss R., J. Physique 38 (1977) 353 